\title{
PET imaging of noradrenaline transporters in Parkinson's disease: focus on scan time
}

\author{
Joachim Brumberg ${ }^{1}$ Johannes Tran-Gia ${ }^{1}$. Constantin Lapa ${ }^{1} \cdot$ Ioannis U. Isaias ${ }^{2} \cdot$ Samuel Samnick $^{1}$
}

Received: 1 October 2018 / Accepted: 1 October 2018 / Published online: 6 October 2018

(c) The Author(s) 2018

\begin{abstract}
Objective In subjects with idiopathic Parkinson's disease (PD) the functional state of the locus coeruleus and the subtle derangements in the finely tuned dopamine-noradrenaline interplay are largely unknown. The PET ligand $(S, S)-\left[{ }^{11} \mathrm{C}\right]-O$-methylreboxetine (C-11 MRB) has been described to reliably bind noradrenaline transporters but long scanning protocols might hamper its use, especially in patients with PD. We aimed to assess the feasibility of reducing C-11 MRB scans to 30 min.

Methods Ten patients with idiopathic PD underwent dynamic C-11 MRB PET (120 min duration) and brain magnetic resonance imaging. Model-based (i.e., simplified and multilinear reference tissue model 2) non-displaceable binding potentials (BP) of selected brain regions were analyzed for a $90 \mathrm{~min}$ scan protocol and compared with BP derived from static 30-min data with different starting times $(30,40,50$ and $60 \mathrm{~min})$ after C-11 MRB injection. Intraclass correlation coefficient and linear regression analysis were used to explore the association between BP of different scan durations. Spearman's $\rho$ served to describe the correlation of BP with demographic and clinical parameters.

Results With respect to kinetic models, $\mathrm{BP}_{50-80}$ and $\mathrm{BP}_{60-90}$ showed the best correlation in several brain areas $\left(R^{2}\right.$ range 0.95-98; $p<0.001)$. The thalamus showed the highest BP on average. No correlation between BP, clinical and demographic characteristics was observed.

Conclusions An acquisition time of $30 \mathrm{~min}$, starting 50 or $60 \mathrm{~min}$ after C-11 MRB injection, allows a reliable estimation of noradrenaline transporter binding values in Parkinsonian people. A short acquisition time can significantly reduce the discomfort of Parkinsonian patients and facilitate PET studies, especially in the medication-off-state.
\end{abstract}

Keywords Parkinson's disease $\cdot$ Locus coeruleus $\cdot$ Noradrenaline transporter $\cdot$ Positron emission tomography

\section{Introduction}

Parkinson's disease (PD) has been historically identified as a nigrostriatal dopamine deficiency syndrome. The dramatic response of most motor and some non-motor symptoms to dopaminergic therapies, coupled with milestone achievements in the understanding of the dysfunction of

Ioannis U. Isaias and Samuel Samnick contributed equally to this work.

Ioannis U. Isaias

Isaias_I@ukw.de

1 Department of Nuclear Medicine, University Hospital and Julius-Maximilians-University, Oberdürrbacher Straße 6, 97080 Würzburg, Germany

2 Department of Neurology, University Hospital and Julius-Maximilians-University, Josef-Schneider-Straße 11, 97080 Würzburg, Germany dopamine homeostasis, furthered this belief [1-3]. However, dopamine neurotransmission may not be the first nor the major neurotransmitter casualty in the pathophysiology of PD [4]. In this respect, there is increasing awareness of variable derangements of non-dopaminergic modulatory systems (e.g., noradrenaline, acetylcholine, and glutamate). However, the general belief is still that the symptoms of PD parallel a neurodegenerative process $[4,5]$, although certain symptoms relate more to adaptive changes and compensatory adjustments than to slowly progressive dopaminergic failure. As an example, levodopa-related dyskinesia might be due to aberrant release of dopamine from serotonin neurons [6], or preserved striatal cholinergic functions [7]. We also advanced the hypothesis that parkinsonian tremor could result from increased noradrenergic activity arising from the locus coeruleus (LC) [8], which could also be responsible for the benign progression of $\mathrm{PD}$ in patients with tremor, owing to a putative neuroprotective and compensatory activity of 
noradrenaline on its target neurons (including the substantia nigra and the striatum) $[9,10]$. This hypothesis has recently been supported by a preliminary study in PD patients with positron emission tomography (PET) using $(S, S)-{ }^{11} \mathrm{C}-2-(\alpha-$ (2-methoxyphenoxy)benzyl)morpholine, also called $(S, S)$ $\left[{ }^{11} \mathrm{C}\right]-O$-methylreboxetine (C-11 MRB), a selective ligand for noradrenaline reuptake transporters (NET), which are localized on noradrenergic synaptic terminals [11]. Information on the noradrenergic terminal density in people with PD is largely missing and our knowledge derives from histopathological examinations, mostly performed in advanced stages of the disease, when compensatory mechanisms are eventually exhausted. Molecular imaging studies in vivo are needed, but have long been hampered by the lack of suitable radioligands. One imaging study used the tracer C-11 RTI-32 to specifically address the noradrenergic activity of the central nervous system in PD patients. The authors showed reduced catecholamine transporter density in the LC and in several regions of the limbic system in depressed PD patients [12]. Recently, Nahimi et al. [11] evaluated the suitability of the ligand C-11 MRB for use in PD patients and observed decreased binding in the thalamus and nucleus ruber, particularly in akinetic-rigid dominant PD patients. Furthermore, a widespread reduced binding of C-11 MRB was found in PD patients with REM sleep behavior disorders, which correlated with the amount of REM sleep without atonia [13]. C-11 MRB was developed by Ding et al. [14, 15] for the exploration of NET availability in patients with psychiatric disorders [16-18]. The main practical limitation of C-11 MRB is the long scan duration; the published scanning protocols comprise dynamic PET scans over at least 90 min [11, 17, 18]. Long protocols, however, cause major discomfort for patients with PD, especially when pausing dopaminergic medication. Arterial input function measurements, to quantify the volume of distribution, could be less of a problem by replacing with the simplified reference tissue model (SRTM) 2 [19] or Ichise's multilinear reference tissue model (MRTM) 2 [20, 21]. These models, as well as the use of the caudate or the occipital cortex as possible reference regions, have recently been validated in people with PD [11]. The aim of this study was to investigate the validity of shorter scanning protocols for C-11 MRB PET in PD patients. We compared non-displaceable binding potentials (BP) of cortical and subcortical brain regions estimated using a 30-min scan duration that started between 30 and 60 min post-injection, with the model-derived binding values obtained by SRTM2 and MRTM2. Furthermore, we tested region-specific BP for correlations with demographic and clinical parameters.

\section{Materials and methods}

\section{Patients}

We consecutively recruited eight men and two women with a diagnosis of PD according to the UK Brain Bank Clinical Diagnostic criteria for PD. Disease severity and stage was assessed with the Unified Parkinson's Disease Rating Scale (UPDRS) and the Hoehn and Yahr (H/Y) scale. Patients were clinically investigated in the morning after overnight withdrawal ( $>12 \mathrm{~h}$ ) of all dopaminergic drugs (meds-off) and at $1 \mathrm{~h}$ (meds-on) after oral intake of 200/50 mg fast-release soluble levodopa/benserazide. Clinical and demographic data are listed in Table 1. Patients with significant comorbidity, previous history of other neurological conditions (e.g., stroke and epilepsy), dementia, depression or sleep disorders (e.g., REM sleep behavior disorder) were not enrolled. None of the patients were taking antipsychotics, antidepressants, or other

Table 1 Patient characteristics

\begin{tabular}{|c|c|c|c|c|c|c|c|}
\hline Patient no. & Sex & Age (years) & DD (years) & $\mathrm{H} / \mathrm{Y}$ (stage) & $\begin{array}{l}\text { UPDRS III } \\
\text { meds-off } \\
\text { (score) }\end{array}$ & $\begin{array}{l}\text { UPDRS III } \\
\text { meds-on } \\
\text { (score) }\end{array}$ & LEDD (mg) \\
\hline 1 & M & 59 & 16 & 2 & 30 & 13 & 405 \\
\hline 2 & M & 59 & 3 & 1 & 14 & 3 & 408 \\
\hline 3 & M & 73 & 2 & 3 & 28 & 10 & 450 \\
\hline 4 & M & 65 & 6 & 3 & 36 & 19 & 720 \\
\hline 5 & $\mathrm{~F}$ & 54 & 2 & 1 & 11 & 6 & 260 \\
\hline 6 & M & 53 & 4 & 1 & 22 & 15 & 557 \\
\hline 7 & F & 61 & 3 & 1 & 25 & 18 & 450 \\
\hline 8 & M & 53 & 3 & 2 & 20 & 16 & 720 \\
\hline 9 & M & 64 & 2 & 1 & 11 & 8 & 780 \\
\hline 10 & M & 64 & 3 & 1 & 15 & 9 & 310 \\
\hline Mean \pm SD & & $60.5 \pm 6.3$ & $4.4 \pm 4.2$ & $1.6 \pm 0.8$ & $21 \pm 8.5$ & $8.3 \pm 7.1$ & $50 \pm 181$ \\
\hline
\end{tabular}

$D D$ disease duration, $H / Y$ Hoehn and Yahr stage, UPDRS Unified Parkinson's Disease Rating Scale, $L E D D$ levodopa-equivalent daily dose 
drugs possibly affecting the noradrenergic system. C-11 MRB was administered on a compassionate use basis in compliance with $\S 37$ of the Declaration of Helsinki and The German Medicinal Products Act, AMG §13.2b. MRI was performed as part of the clinical work-up. The study was approved by the local institutional review board (IRB approval: 2017040601). All patients gave written informed consent to the diagnostic procedures.

\section{Preparation of C-11 MRB}

$(2 S, 3 S)$-Desethylreboxetine used as precursor for radiolabeling and the MRB reference were purchased from ABX (Radeberg, Germany). All solvents and reagents were provided in Ph. Eur. quality from Merck KG (Darmstadt, Germany) or AppliChem GmbH (Darmstadt, Germany).

C-11 MRB was synthesized on a Tracerlab-Fx-C-Pro synthesis unit (GE Medical Systems, Uppsala, Sweden) following the method published previously by Ding et al., with minor modifications $[18,22]$. The radiochemical yield was typically between 4.5 and $5.5 \mathrm{GBq}$ per batch with a specific activity, as determined by analytical HPLC and MRB reference, of $40-60 \mathrm{GBq} / \mu \mathrm{mol}(\mathrm{EOS})$ in a total synthesis time of $45 \mathrm{~min}$.

\section{PET scans}

PET scans were obtained with a combined PET/CT scanner (Biograph mCT 64; Siemens Healthineers, Knoxville, TN, USA). Prior to the acquisition, an intravenous catheter was placed in the antecubital vein of the left or right arm. The patients were positioned comfortably in the PET scanner and instructed to remain still for the total duration of the scan. The patient's head was fixed in a head holder to avoid motion artefacts. After the acquisition of a low-dose CT for attenuation correction (CARE Dose 4D, $80 \mathrm{mAs}$, $120 \mathrm{kV}$; matrix: $512 \times 512$; 2 -mm slice thickness; increment: $30 \mathrm{~mm} / \mathrm{s}$; rotation time: $0.5 \mathrm{~s}$; pitch index: 0.8 ), C-11 MRB was administered intravenously as a slow manual bolus injection of $635 \pm 29 \mathrm{MBq}$. Simultaneously with the injection, a dynamic PET acquisition with $120 \mathrm{~min}$ duration was initiated using the 3D list mode. After the acquisition, the data were binned in 33 frames of progressively longer durations $(6 \times 30 \mathrm{~s}, 3 \times 60 \mathrm{~s}, 2 \times 120 \mathrm{~s}$, $22 \times 300 \mathrm{~s}$ ), and PET images were reconstructed iteratively (HD-PET, 24 subsets, 3 iterations; Gaussian filtering: $5.0 \mathrm{~mm}$; matrix: $400 \times 400$; axial resolution: $2.0 \mathrm{~mm}$; inplane resolution: $2.04 \times 2.04 \mathrm{~mm}^{2}$ ). Acquisition and data reconstruction were performed using dedicated manufacturer software (syngo MI.PET/CT; Siemens Healthineers, Erlangen, Germany).

\section{MR imaging}

Magnetic resonance imaging (MRI) was performed on a 3T whole-body scanner (MAGNETOM Trio; Siemens Healthineers, Erlangen, Germany) equipped with a 12-channel, phased-array head coil for signal reception. In all subjects, a standard cranial MRI protocol was applied, including an isotropic high-resolution structural T1-weighted MPRAGE-Sequence (Turbo-FLASH $1.0 \times 1.0 \times 1.0 \mathrm{~mm}^{3}$, TR $2530 \mathrm{~ms}$, TE $3.37 \mathrm{~ms}$, FA $9^{\circ}$, TI $1200 \mathrm{~ms}$ ) for anatomical co-registration with the PET images.

\section{Image analysis}

\section{Data preprocessing}

All image data were processed and analyzed using PMOD image analysis software version 3.7 (PMOD Technologies Ltd, Zurich, Switzerland). Dynamic PET data were motion-corrected to a late time point image [40-45 $\mathrm{min}$ post-injection (p.i.)] of the scan. An average PET image of 20-60 min p.i. of the motion-corrected time series was calculated for each subject. Based on this, the dynamic PET scan was registered and matched to the individuals' MP-RAGE image volume. Furthermore, four static PET images of $30 \mathrm{~min}$ scan time were created for each patient by averaging over six consecutive of these preprocessed frames starting at 30, 40, 50 and $60 \mathrm{~min}$.

\section{Vol definition}

Three probability maps were obtained, based on the individuals' MP-RAGE sequence, by applying the segmentation method [23]. Normalization of the MR images was performed via probability maps transformation into the standard anatomical space of the Montreal Neurological Institute (MNI) [24]. Based on the N30R83 maximum probability atlas by Hammers and colleagues [25], 17 target volumes of interest (VOIs) were defined: amygdala (left/right; $1 / \mathrm{r}$ ), brainstem, caudate nucleus (1/r), cerebellum $(1 / r)$, frontal lobe $(1 / r)$, hippocampus $(1 / r)$, parietal lobe $(1 / \mathrm{r})$, putamen $(1 / \mathrm{r})$ and thalamus $(1 / \mathrm{r})$. Two additional atlas-based auxiliary VOIs were created, one containing the regions of the occipital lobe, and a second by merging the left and right thalamus. The transformation between the patient brain and the MNI space was then used to inversely transform the VOIs to the individuals' MRI and the co-registered PET space. Last, a VOI containing the LC was outlined using previously published atlas coordinates [26]. 


\section{Kinetic modeling}

VOI-specific time activity curves (TAC) from 0 to $90 \mathrm{~min}$ p.i. were generated from the dynamic PET data for each patient. To determine the efflux rate constant $\left(k_{2}^{\prime}\right)$, oneparameter MRTM was applied for each of these TACs using the fused thalamus VOI as the transporter-rich region and the occipital lobe as the reference region. Then, the mean $k_{2}^{\prime}$ of all ten patients was calculated and transferred to the twoparameter MRTM2 and SRTM2 (20) to determine binding values $\left(\mathrm{BP}_{\mathrm{MRTM} 2}\right.$ and $\left.\mathrm{BP}_{\mathrm{SRTM} 2}\right)$ of the eighteen target VOIs for each patient. Additionally, BP of the four static brain PETs was calculated for each of the above regions using average regional uptake values $[\mathrm{BP}=$ (mean activity concentration VOI - mean activity concentration occipital cortex $) /($ mean activity concentration occipital cortex)]. 30-min time frames starting at four different times p.i. were used $\left(\mathrm{BP}_{30-60}, \mathrm{BP}_{40-70}, \mathrm{BP}_{50-80}, \mathrm{BP}_{60-90}\right)$.

\section{Statistical analysis}

Clinical variables and BP estimates were tested for normality using the Shapiro-Wilk test. We calculated (region-wise) the Spearman's rank correlation coefficient (Spearman's $\rho$ ) and the intraclass correlation coefficient (ICC) between $\mathrm{BP}_{30-60}, \mathrm{BP}_{40-70}, \mathrm{BP}_{50-80}$ or $\mathrm{BP}_{60-90}$ and the model-based binding values $\left(\mathrm{BP}_{\mathrm{MRTM} 2}\right.$ and $\left.\mathrm{BP}_{\mathrm{SRTM} 2}\right)$. A linear regression analysis was used to describe the correlation between binding values provided by the four static 30-min brain PETs and the two model-derived $\mathrm{BP}_{\mathrm{MRTM} 2}$ and $\mathrm{BP}_{\mathrm{SRTM} 2}$ (all regions). The association between the binding value of the thalamus with age and clinical variables [i.e., disease duration, $\mathrm{H} / \mathrm{Y}$ stage, UPDRS-III score, and levodopa-equivalent daily dose (LEDD)] was evaluated with a multivariate analysis of variance and Spearman's $\rho$. Of note, we assumed no relevant influence of laterality on time-dependent BP and we pooled left and right hemisphere for all paired brain regions. Significance was set at $p<0.05$ and corrected for multiple comparisons.

\section{Results}

Region-wise correlational analyses are listed in Tables 2 and 3. The linear regression analysis is shown in Figs. 1 and 2. With respect to the model-derived binding values, $\mathrm{BP}_{60-90}\left(\mathrm{BP}_{60-90}=0.97 \times \mathrm{BP}_{\mathrm{SRTM} 2}-0.01, R^{2}=0.97\right.$, $p<0.001 ; \mathrm{BP}_{60-90}=0.96 \times \mathrm{BP}_{\mathrm{MRTM} 2}-0.01, R^{2}=0.96$, $p<0.00)$ and $\mathrm{BP}_{50-80}\left(\mathrm{BP}_{50-80}=0.93 \times \mathrm{BP}_{\mathrm{SRTM} 2}-0.01\right.$, $R^{2}=0.96, p<0.001 ; \mathrm{BP}_{50-80}=0.90 \times \mathrm{BP}_{\mathrm{MRTM} 2}-0.02$, $\left.R^{2}=0.95, p<0.001\right)$ showed a very high correlation. Less significant prediction values were observed for $\mathrm{BP}_{40-70}$ $\left(\mathrm{BP}_{40-70}=0.87 \times \mathrm{BP}_{\mathrm{SRTM} 2}-0.02, R^{2}=0.95, p<0.001\right.$;

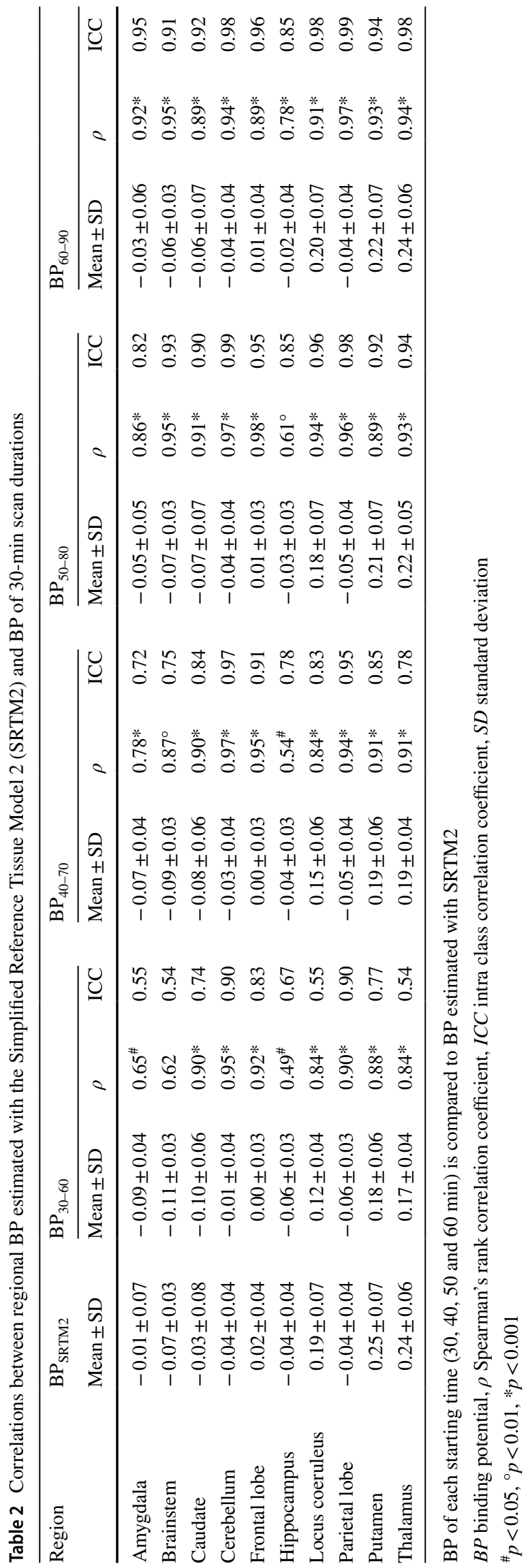


$\left.\mathrm{BP}_{40-70}=0.83 \times \mathrm{BP}_{\mathrm{MRTM} 2}-0.03, R^{2}=0.91, p<0.001\right)$ and $\mathrm{BP}_{30-60}\left(\mathrm{BP}_{30-60}=0.81 \times \mathrm{BP}_{\mathrm{SRTM}_{2}}-0.03, R^{2}=0.90\right.$, $p<0.001 ; \mathrm{BP}_{30-60}=0.77 \times \mathrm{BP}_{\mathrm{MRTM} 2}-0.04, R^{2}=0.84$, $p<0.001)$. The highest average $\mathrm{BP}$ value was found in the thalamus, followed by the putamen and the LC. The average thalamic NET binding potential ranged between 0.22 and 0.27 for $\mathrm{BP}_{\mathrm{MRTM} 2}, \mathrm{BP}_{\mathrm{SRTM} 2}, \mathrm{BP}_{50-80}$ and $\mathrm{BP}_{60-90}$, whereas it was lower for $\mathrm{BP}_{30-60}$ and $\mathrm{BP}_{40-70}(0.17$ and 0.19). No significant correlations between BP, demographic, and clinical parameters were found.

\section{Discussion}

This study suggests that an acquisition time of $30 \mathrm{~min}$ for C-11 MRB PET can offer a reliable estimation of cerebral NET density. This information is of relevance to reduce the scan duration and therefore the discomfort of Parkinsonian patients.

Acquisitions starting at 50 and $60 \mathrm{~min}$ showed higher correlation values regarding $\mathrm{BP}_{\mathrm{SRTM} 2}$ and $\mathrm{BP}_{\mathrm{MRTM} 2}$ than the scans starting at 30 and 40 min p.i. (Figs. 1, 2). This finding suggests that for C-11 MRB, many cortical and subcortical regions reach a stable equilibrium at later time points of the scan. An early start combined with a short acquisition duration might partially capture the tracer distribution before the equilibrium has been reached. Additionally, it might not even cover the phase of stable equilibrium occurring at later time points. Accordingly, scans of 30 min duration starting at a later time point ( 50 or $60 \mathrm{~min}$ ) showed better ICC values for all tested brain regions (Tables 2, 3). These results are in line with previous PET studies on the noradrenergic system showing stable TACs for the thalamus, the basal ganglia, the cerebellum, and cortical regions between 50 and 90 min p.i. using C-11 MRB [27], C-11 MENET [28], or F-18 FMeNER-D 2 [29].

Previous studies in non-human primates and humans tested the caudate nucleus, the putamen, and the occipital cortex as potential reference regions for C-11 MRB [30, 31]. In PD patients, only the caudate and the occipital cortex have been validated for this ligand, with the latter showing slightly lower BP [11]. Besides methodological issues [21], we chose the occipital cortex as a reference because of the important role of the caudate nucleus in the pathophysiology of PD. Motor PD symptoms appear when the loss of putamen dopamine level reaches $70-80 \%$, which corresponds to a 50-60\% decrease in dopamine neurons $[32,33]$. The caudate nucleus is instead relatively spared at early stages of PD [34] and represents a possible site of still-active compensatory and neuroprotective mechanisms upon motor symptoms onset [7], also involving the noradrenergic system arising from the LC [35]. 

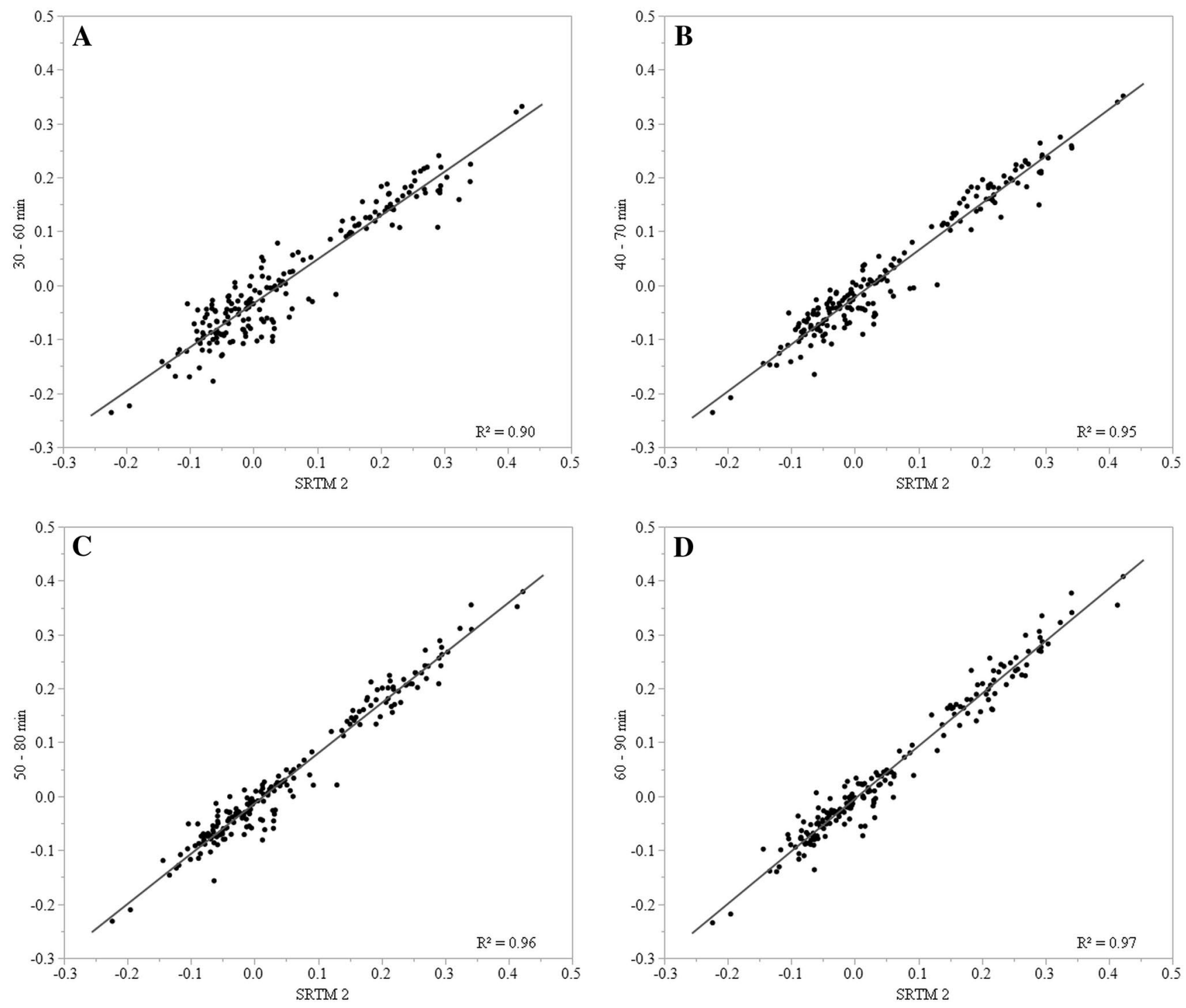

Fig. 1 Scatter plots and linear regression analysis of statically derived binding potentials (BP) and binding values estimated with the Simplified Reference Tissue Model 2 (SRTM2). The upper row shows the $\mathrm{BP}$ of 30-min scan durations with acquisition start at $30 \mathrm{~min}$ (a) and

The parameter $k_{2}^{\prime}$ is the clearance rate constant from the reference region to plasma, and needs to be defined a priori for the reference methods SRTM2 and MRTM2 [20]. This parameter can be estimated for each subject by applying the three-parameter model MRTM to the individual TAC of a high-binding region and a reference region (in our case, the thalamus and the occipital brain area, respectively). In the cohort of this study, an average $k_{2}^{\prime}$ of $0.0322 \pm 0.0105 \mathrm{~min}^{-1}$ was determined. It is in the range of previously measured values of $k_{2}^{\prime}$ based on one-tissue compartment modeling with arterial input measurements in PD patients and healthy controls [11].

$40 \mathrm{~min}$ (b), the lower row shows BP starting at $50 \mathrm{~min}$ (c) and $60 \mathrm{~min}$ (d). All comparisons were plotted on BP estimated with the SRTM2. Regression lines are depicted in grey

Finally, with regard to the actual NET measurements, the $\mathrm{BP}_{50-80}$ and $\mathrm{BP}_{60-90}$ shown in this study (Tables 2, 3) are in line with previous reports $[11,13,18,28,29]$. Multivariate analysis of variance showed no significant correlation between any BP of noradrenaline transporters and demographic or clinical measures (i.e., disease duration, disease severity and LEDD) in all brain regions investigated. These findings are in line with studies showing a negative correlation between age and BP of the thalamus and LC only in young healthy subjects (mean age: 35 years) [18], but not in the elderly (mean age: 66 years) and PD patients [11]. The small sample size of our study population, as well as 

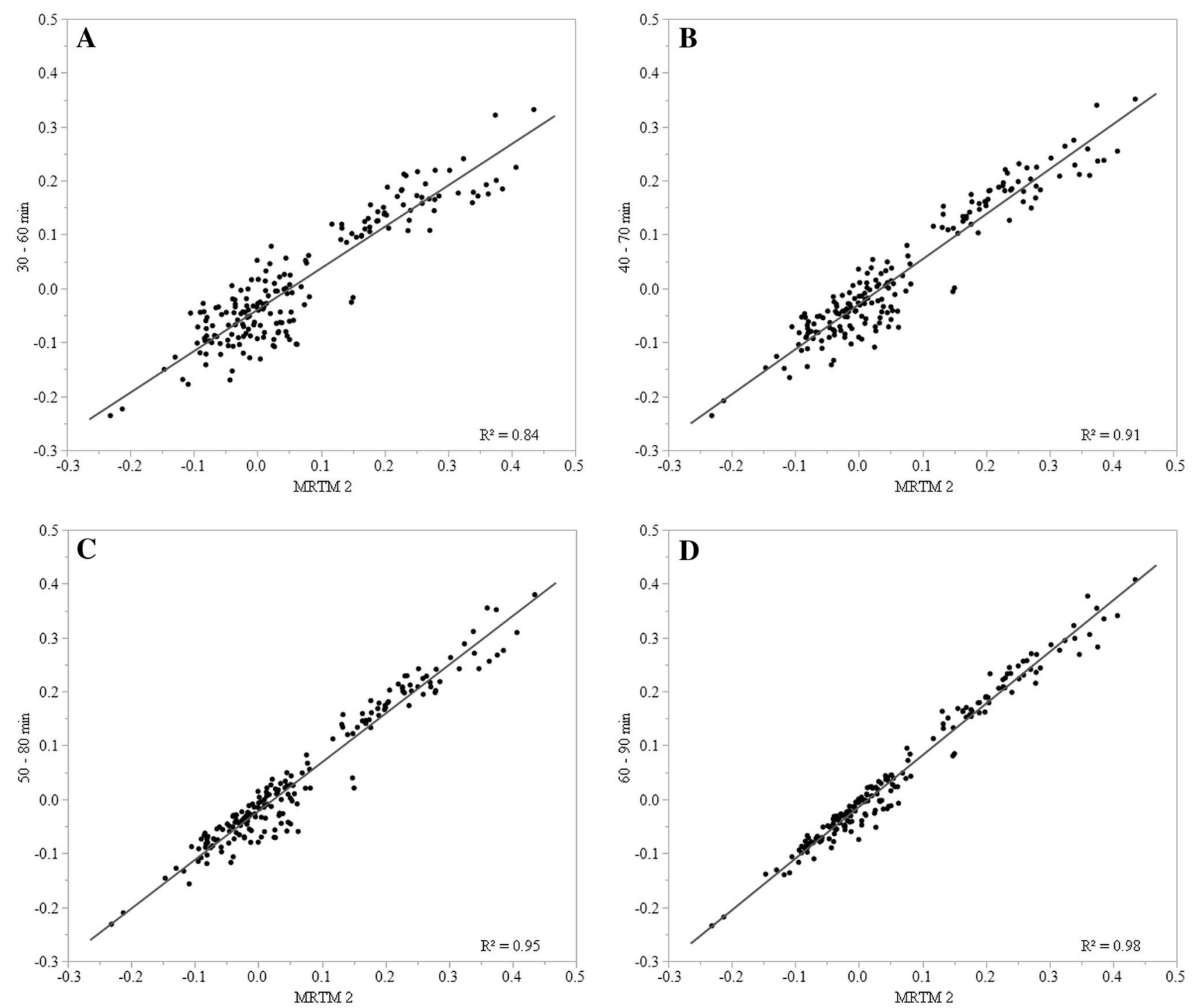

Fig. 2 Scatter plots and linear regression analysis of statically derived binding potentials (BP) and binding values estimated with Ichise's Multilinear Reference Tissue Model 2 (MRTM2). The upper row shows the BP of 30-min scan durations with acquisition start at

$30 \mathrm{~min}$ (a) and $40 \mathrm{~min}$ (b), the lower row shows BP starting at $50 \mathrm{~min}$ (c) and $60 \mathrm{~min}$ (d). All comparisons were plotted on BP estimated with the MRTM2. Regression lines are depicted in grey

the results of previous studies, however, prevent any firm conclusion on demographic and clinical correlations of C-11 MRB binding values. In our case, while disease severity and LEDD were reasonably well represented in our study cohort (Table 1), all but two subjects had disease duration below 5 years and only two females were included in this study. Furthermore, no optical tracking system for motion correction was available, which can considerably improve the analysis of small brain areas such as the LC. Nevertheless, also in agreement with Nahimi et al. [11], it is of relevance to note that C-11 MRB binding was not influenced by the dosage of dopaminergic drugs (i.e., LEDD). This result also parallels previous findings on dopamine reuptake transporter

binding values measured with I-123 FP-CIT and SPECT [36]. Further studies are warranted to deepen our understanding of the noradrenergic system in people with PD, with emphasis on clinical phenotypes (e.g., tremor-dominant PD) [37], compensatory and protective mechanisms [8-10].

Funding The study was sponsored in part by the "Interdisziplinäres Zentrum für Klinische Forschung" of the University Hospital Würzburg and by the "Fondazione Grigioni per il Morbo di Parkinson".

\section{Compliance with ethical standards}

Conflict of interest The authors declare that they have nothing to disclose. 
Open Access This article is distributed under the terms of the Creative Commons Attribution 4.0 International License (http://creativeco mmons.org/licenses/by/4.0/), which permits unrestricted use, distribution, and reproduction in any medium, provided you give appropriate credit to the original author(s) and the source, provide a link to the Creative Commons license, and indicate if changes were made.

\section{References}

1. Carlsson A, Lindqvist M, Magnusson T. 3,4-Dihydroxyphenylalanine and 5-hydroxytryptophan as reserpine antagonists. Nature. 1957;180:1200.

2. Cotzias GC. L-Dopa for parkinsonism. N Engl J Med. 1968;278:630.

3. Benamer HT, Patterson J, Wyper DJ, Hadley DM, Macphee GJ, Grosset DG. Correlation of Parkinson's disease severity and duration with 123I-FP-CIT SPECT striatal uptake. Mov Disord. 2000;15:692-8.

4. Braak H, Del Tredici K. Cortico-basal ganglia-cortical circuitry in Parkinson's disease reconsidered. Exp Neurol. 2008;212:226-9.

5. Espay AJ, LeWitt PA, Kaufmann H. Norepinephrine deficiency in Parkinson's disease: the case for noradrenergic enhancement. Mov Disord. 2014;29:1710-9.

6. Cenci MA. Presynaptic mechanisms of 1-DOPA-induced dyskinesia: the findings, the debate, and the therapeutic implications. Front Neurol. 2014;5:242.

7. Brumberg J, Küsters S, Al-Momani E, Marotta G, Cosgrove KP, van Dyck $\mathrm{CH}$, et al. Cholinergic activity and levodopa-induced dyskinesia: a multitracer molecular imaging study. Ann Clin Transl Neurol. 2017;4:632-9.

8. Isaias IU, Marzegan A, Pezzoli G, Marotta G, Canesi M, Biella $\mathrm{G}$, et al. A role for locus coeruleus in Parkinson tremor. Front Hum Neurosci. 2012;3:179.

9. Rommelfanger KS, Weinshenker D. Norepinephrine. The redheaded stepchild of Parkinson's disease. Biochem Pharmacol. 2007;74:177-90.

10. Isaias IU, Marotta G, Pezzoli G, Sabri O, Schwarz J, Crenna P, et al. Enhanced catecholamine transporter binding in the locus coeruleus of patients with early Parkinson disease. BMC Neurol. 2011. https://doi.org/10.1186/1471-2377-11-88.

11. Nahimi A, Sommerauer M, Kinnerup MB, Østergaard K, Winterdahl M, Jacobsen $\mathrm{J}$, et al. Noradrenergic deficits in Parkinson's disease imaged with 11C-MeNER. J Nucl Med. 2018;59:659-64.

12. Remy P, Doder M, Lees A, Turjanski N, Brooks D. Depression in Parkinson's disease: loss of dopamine and noradrenaline innervation in the limbic system. Brain. 2005;128:1314-22.

13. Sommerauer M, Fedorova TD, Hansen AK, Knudsen K, Otto $\mathrm{M}$, Jeppesen J, et al. Evaluation of the noradrenergic system in Parkinson's disease: an 11C-MeNER PET and neuromelanin MRI study. Brain. 2018;141:496-504.

14. Ding YS, Lin KS, Garza V, Carter P, Alexoff D, Logan J, et al. Evaluation of a new norepinephrine transporter PET ligand in baboons, both in brain and peripheral organs. Synapse. 2003;50:345-52.

15. Ding YS, Lin KS, Logan J, Benveniste H, Carter P. Comparative evaluation of positron emission tomography radiotracers for imaging the norepinephrine transporter: $(S, S)$ and $(R, R)$ enantiomers of reboxetine analogs $([11 \mathrm{C}]$ methylreboxetine, 3-Cl[11C]methylreboxetine and [18F]fluororeboxetine), $(R)-[11 \mathrm{C}]$ nisox. J Neurochem. 2005;94:337-51.
16. Pietrzak RH, Gallezot JD, Ding YS, Henry S, Potenza MN, Southwick SM, et al. Association of posttraumatic stress disorder with reduced in vivo norepinephrine transporter availability in the locus coeruleus. JAMA Psychiatry. 2013;70:1199-205.

17. Hesse S, Becker GA, Rullmann M, Bresch A, Luthardt J, Hankir MK, et al. Central noradrenaline transporter availability in highly obese, non-depressed individuals. Eur J Nucl Med Mol Imaging. 2017;44:1056-64.

18. Ding YS, Singhal T, Planeta-Wilson B, Gallezot JD, Nabulsi $\mathrm{N}$, Labaree D, et al. PET imaging of the effects of age and cocaine on the norepinephrine transporter in the human brain using $(S, S)-[(11) \mathrm{C}] O$-methylreboxetine and HRRT. Synapse. 2010;64:30-8.

19. $\mathrm{Wu} \mathrm{Y,} \mathrm{Carson} \mathrm{RE.} \mathrm{Noise} \mathrm{reduction} \mathrm{in} \mathrm{the} \mathrm{simplified} \mathrm{reference}$ tissue model for neuroreceptor functional imaging. J Cereb Blood Flow Metab. 2002;22:1440-52.

20. Ichise M, Liow JS, Lu JQ, Takano A, Model K, Toyama H, et al. Linearized reference tissue parametric imaging methods: application to [11C]DASB positron emission tomography studies of the serotonin transporter in human brain. J Cereb Blood Flow Metab. 2003;23:1096-112.

21. Ding YS. Progress in PET imaging of the norepinephrine transporter system. In: Dierckx RAJO, Otte A, de Vries EFJ, van Waarde A, Leenders KL, editors. PET and SPECT of neurobiological systems. Heidelberg: Springer; 2014. pp. 561-84.

22. Lin KS, Ding YS. Synthesis, enantiomeric resolution, and selective C-11 methylation of a highly selective radioligand for imaging the norepinephrine transporter with positron emission tomography. Chirality. 2004;16:475-81.

23. Ashburner J, Friston KJ. Unified segmentation. Neuroimage. 2005;26:839-51.

24. Collins DL, Zijdenbos AP, Kollokian V, Sled JG, Kabani NJ, Holmes CJ,et al. Design and construction of a realistic digital brain phantom. IEEE Trans Med Imaging. 1998;17:463-8.

25. Hammers A, Allom R, Koepp MJ, Free SL, Myers R, Lemieux $\mathrm{L}$, et al. Three-dimensional maximum probability atlas of the human brain, with particular reference to the temporal lobe. Hum Brain Mapp. 2003;19:224-47.

26. Keren NI, Lozar CT, Harris KC, Morgan PS, Eckert MA. In vivo mapping of the human locus coeruleus. Neuroimage. 2009;47:1261-7.

27. Logan J, Wang GJ, Telang F, Fowler JS, Alexoff D, Zabroski J, et al. Imaging the norepinephrine transporter in humans with $(S, S)$-[11C] $O$-methyl reboxetine and PET: problems and progress. Nucl Med Biol. 2007;34:667-79.

28. Adhikarla V, Zeng F, Votaw JR, Goodman MM, Nye JA. Compartmental modeling of [(11)C]MENET binding to the norepinephrine transporter in the healthy human brain. Nucl Med Biol. 2016;43:318-23.

29. Moriguchi S, Kimura Y, Ichise M, Arakawa R, Takano H, Seki C, et al. PET Quantification of the norepinephrine transporter in human brain with $(S, S)$-18F-FMeNER-D2. J Nucl Med. 2017;58:1140-5.

30. Gallezot JD, Weinzimmer D, Nabulsi N, Lin SF, Fowles K, Sandiego C, et al. Evaluation of [(11)C]MRB for assessment of occupancy of norepinephrine transporters: studies with atomoxetine in non-human primates. Neuroimage. 2011;56:268-79.

31. Hannestad J, Gallezot JD, Planeta-Wilson B, Lin SF, Williams WA, van Dyck $\mathrm{CH}$, et al. Clinically relevant doses of methylphenidate significantly occupy norepinephrine transporters in humans in vivo. Biol Psychiatry. 2010;68:854-60.

32. Fearnley JM, Lees AJ. Ageing and Parkinson's disease: substantia nigra regional selectivity. Brain. 1991;114:2283-301.

33. de la Fuente-Fernández R, Schulzer M, Kuramoto L, Kuramoto L, Cragg J, Ramachandiran N, Au WL, et al. Age-specific 
progression of nigrostriatal dysfunction in Parkinson's disease. Ann Neurol. 2011;69:803-10.

34. Isaias IU, Benti R, Cilia R, Canesi M, Marotta G, Gerundini $\mathrm{P}$, et al. [123I]FP-CIT striatal binding in early Parkinson's disease patients with tremor vs. akinetic-rigid onset. Neuroreport. 2007; 18:1499-502.

35. Lategan AJ, Marien MR, Colpaert FC. Effects of locus coeruleus lesions on the release of endogenous dopamine in the rat nucleus accumbens and caudate nucleus as determined by intracerebral microdialysis. Brain Res. 1990;523:134-8.
36. Booij J, Kemp P. Dopamine transporter imaging with [(123)I] FP-CIT SPECT: potential effects of drugs. Eur J Nucl Med Mol Imaging. 2008;35:424-38.

37. Ghaemi M, Raethjen J, Hilker R, Rudolf J, Sobesky J, Deuschl G, et al. Monosymptomatic resting tremor and Parkinson's disease: a multitracer positron emission tomographic study. Mov Disord. 2002;17:782-8. 